\title{
Electron Waiting Times of a Cooper Pair Splitter
}

\author{
Walldorf, Nicklas; Padurariu, Ciprian; Jauho, Antti-Pekka; Flindt, Christian
}

Published in:

Physical Review Letters

Link to article, DOI:

10.1103/PhysRevLett.120.087701

Publication date:

2018

Document Version

Publisher's PDF, also known as Version of record

Link back to DTU Orbit

Citation (APA):

Walldorf, N., Padurariu, C., Jauho, A-P., \& Flindt, C. (2018). Electron Waiting Times of a Cooper Pair Splitter. Physical Review Letters, 120(8), [087701]. https://doi.org/10.1103/PhysRevLett.120.087701

\section{General rights}

Copyright and moral rights for the publications made accessible in the public portal are retained by the authors and/or other copyright owners and it is a condition of accessing publications that users recognise and abide by the legal requirements associated with these rights.

- Users may download and print one copy of any publication from the public portal for the purpose of private study or research.

- You may not further distribute the material or use it for any profit-making activity or commercial gain

- You may freely distribute the URL identifying the publication in the public portal

If you believe that this document breaches copyright please contact us providing details, and we will remove access to the work immediately and investigate your claim 


\title{
Electron Waiting Times of a Cooper Pair Splitter
}

\author{
Nicklas Walldorf, ${ }^{1}$ Ciprian Padurariu, ${ }^{2,3}$ Antti-Pekka Jauho, ${ }^{1}$ and Christian Flindt ${ }^{2}$ \\ ${ }^{1}$ Center for Nanostructured Graphene (CNG), Department of Micro- and Nanotechnology, \\ Technical University of Denmark, DK-2800 Kongens Lyngby, Denmark \\ ${ }^{2}$ Department of Applied Physics, Aalto University, 00076 Aalto, Finland \\ ${ }^{3}$ Institute for Complex Quantum Systems and IQST, Ulm University, Albert Einstein-Allee 11, 89069 Ulm, Germany
}

(Received 13 September 2017; revised manuscript received 13 December 2017; published 22 February 2018)

\begin{abstract}
Electron waiting times are an important concept in the analysis of quantum transport in nanoscale conductors. Here we show that the statistics of electron waiting times can be used to characterize Cooper pair splitters that create spatially separated spin-entangled electrons. A short waiting time between electrons tunneling into different leads is associated with the fast emission of a split Cooper pair, while long waiting times are governed by the slow injection of Cooper pairs from a superconductor. Experimentally, the waiting time distributions can be measured using real-time single-electron detectors in the regime of slow tunneling, where conventional current measurements are demanding. Our work is important for understanding the fundamental transport processes in Cooper pair splitters and the predictions may be verified using current technology.
\end{abstract}

DOI: 10.1103/PhysRevLett.120.087701

Introduction.-Quantum technologies that exploit nonclassical phenomena such as the discreteness of physical observables, coherent superpositions, and quantum entanglement promise solutions to current challenges in communication, computation, sensing, and metrology [1]. For solid-state quantum computers, an important building block is a device that can generate pairs of entangled electrons [2]. In one prominent approach, Cooper pairs in a superconductor are converted into spatially separated electrons that preserve the entanglement of their spins $[3,4]$. Cooper pair splitters have been realized in architectures based on superconductor-normal-state hybrid systems [5-7], InAs nanowires [8-11], carbon nanotubes [12-16], and recently graphene structures [17-19].

The efficiency of Cooper pair splitters can be determined using conductance measurements [8-17]. For some setups, the efficiency is approaching unity [10,14], indicating that Cooper pair splitters may be suited for electronics-based quantum technologies. One may now hope to detect the entanglement of the outgoing electrons by measuring the cross correlations of the currents in the output channels [10,20-22]. However, while these approaches are based on conventional current measurements, recent progress in the real-time detection of single electrons is opening another promising avenue for understanding quantum transport in nanoscale devices [23].

In this Letter, we propose to characterize Cooper pair splitters using the distribution of electron waiting times. The electron waiting time is the time that passes between subsequent tunneling events. Waiting time distributions (WTDs) have in recent years been investigated theoretically (a)

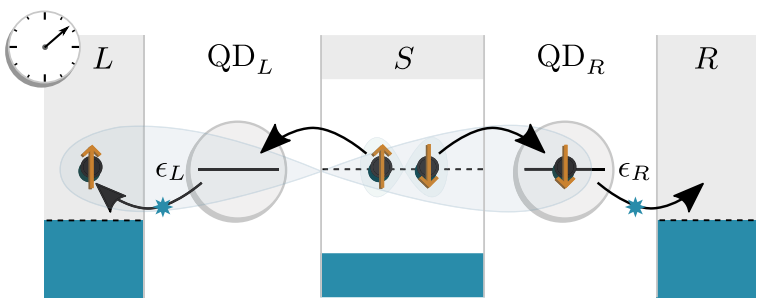

(b)

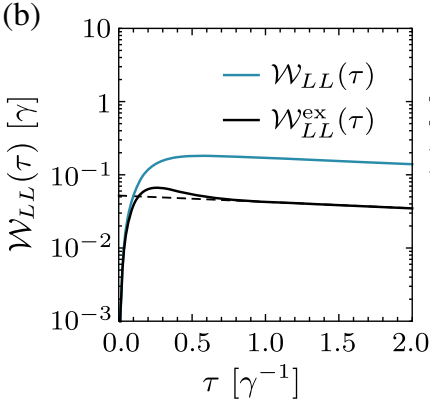

(c)

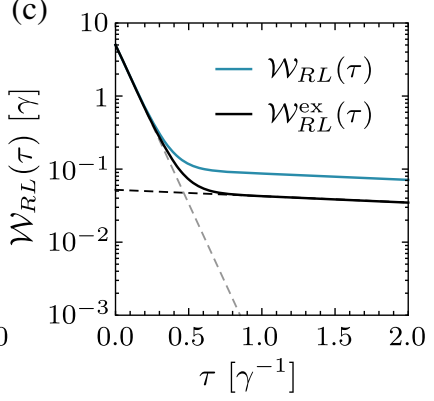

FIG. 1. Electron waiting times of a Cooper pair splitter. (a) Two QDs are coupled to a superconducting source of Cooper pairs and two normal-metal drains. A tunneling event (star) starts the clock, which symbolizes the measurement scheme based on singleelectron detectors [23,52-54]. A subsequent tunneling event stops it. WTDs for tunneling into the same or different leads are shown in (b) and (c). The WTDs $\mathcal{W}_{j i}(\tau)\left[\mathcal{W}_{j i}^{\mathrm{ex}}(\tau)\right]$ are evaluated using Eq. (4) [(5)]. Parameters are $\xi:=\gamma_{L}=\gamma_{R}=10 \gamma$, $\gamma_{\mathrm{CPS}}=\gamma_{\mathrm{EC}}=\gamma$, and $\epsilon_{L}=\epsilon_{R}=0$. Dashed lines are exponentials with decay rates $\xi$ (gray) and $2 \gamma_{\mathrm{CPS}}^{2} / \xi$ (black). Corresponding to the recent experiments, the rate $\gamma$ would be on the order of kilohertz and the waiting times would be in the millisecond range [52-54]. 
for quantum transport in quantum dots [24-38], mesoscopic conductors [39-46], and superconducting devices [47-51]. Moreover, in a very recent experiment, the distribution of electron waiting times was measured for a quantum dot [52]. Here, we show that the WTD is a sensitive tool to unravel the working principle of the Cooper pair splitter in Fig. 1(a). As we discuss below, WTDs such as those in Figs. 1(b) and 1(c) provide clear signatures of the Cooper pair splitting. Specifically, the splitting of Cooper pairs is associated with a large peak at short times in the WTD for tunneling into different drains [Fig. 1(c)]. This information is complementary to what can be learned from conventional current and noise measurements. In addition, with the ability to detect single electrons participating in Andreev tunneling across normal-statesuperconductor interfaces [53,54], a measurement of the electron waiting times in a Cooper pair splitter appears feasible with current technology. In the recent experiment on WTDs, the typical waiting times were on the order of milliseconds [52], which corresponds well to the kilohertz tunneling rates reported in Refs. [53,54]. Importantly, such small tunneling rates do not produce electrical currents that can be measured using standard techniques. On the other hand, the tunneling of electrons can be detected in real time and the distribution of waiting times can be measured.

Cooper pair splitter.-The Cooper pair splitter consists of two quantum dots (QDs) coupled to a superconductor and two normal leads [4]. The grounded superconductor acts as a source of Cooper pairs. The negatively biased leads serve as drains for electrons in the QDs. Coulomb interactions are so strong that each QD cannot be occupied by more than one electron at a time. With a large superconducting gap, we may focus on the subgap transport (the working regime is specified below). The superconductor can then be included in an effective Hamiltonian of the QDs reading [55-61]

$$
\begin{aligned}
\hat{H}_{\mathrm{QDs}}= & \sum_{\ell \sigma} \epsilon_{\ell} \hat{d}_{\ell \sigma}^{\dagger} \hat{d}_{\ell \sigma}-\gamma_{\mathrm{EC}} \sum_{\sigma}\left(\hat{d}_{L \sigma}^{\dagger} \hat{d}_{R \sigma}+\text { H.c. }\right) \\
& -\frac{\gamma_{\mathrm{CPS}}}{\sqrt{2}}\left(\hat{d}_{L \downarrow}^{\dagger} \hat{d}_{R \uparrow}^{\dagger}-\hat{d}_{L \uparrow}^{\dagger} \hat{d}_{R \downarrow}^{\dagger}+\text { H.c. }\right) .
\end{aligned}
$$

Here, the operator $\hat{d}_{\ell \sigma}^{\dagger}\left(\hat{d}_{\ell \sigma}\right)$ creates (annihilates) an electron in $\mathrm{QD}_{\ell}, \ell \in\{L, R\}$ with spin $\sigma \in\{\uparrow, \downarrow\}$ and energy $\epsilon_{\ell}$ relative to the chemical potential of the superconductor, $\mu_{S}=0$. The amplitudes $\gamma_{\mathrm{CPS}}$ and $\gamma_{\mathrm{EC}}$ correspond to Cooper pair splitting (CPS) and elastic cotunneling (EC) processes, respectively, and can be expressed in terms of microscopic parameters following Ref. [55]. We have excluded direct coupling between the QDs as in the experiment of Ref. [17], but such processes can easily be incorporated within our formalism. In the CPS processes, a Cooper pair in the superconductor is converted into two spin-entangled electrons in a singlet state with one electron in each QD or vice versa. Such processes are favored when the empty state of the QDs is energetically degenerate with the doubly occupied state, $\epsilon_{L}+\epsilon_{R}=0$ [62-65]. In the spin-preserving EC processes, an electron in one of the QDs is transferred via the superconductor to the other QD. These processes are on resonance when the QD levels are energetically aligned, $\epsilon_{L}=\epsilon_{R}$.

Transport through each QD is described by resonant tunneling and must be treated to all orders in the coupling to the leads. When the resonant level is deep inside the transport energy window, the transport can be described by a Markovian quantum master equation for the reduced density matrix $\hat{\rho}$ of the QDs (with $\hbar=1$ ) $[55,66]$

$$
\frac{d}{d t} \hat{\rho}=\mathcal{L} \hat{\rho}=-i\left[\hat{H}_{\mathrm{QDs}}, \hat{\rho}\right]+\mathcal{D} \hat{\rho} .
$$

Here, the Liouvillian $\mathcal{L}$ describes both coherent processes governed by $\hat{H}_{\mathrm{QDs}}$ and incoherent single-electron jumps to the normal metals captured by the Lindblad dissipator

$$
\mathcal{D} \hat{\rho}=\sum_{\ell \sigma} \gamma_{\ell}\left[\hat{d}_{\ell \sigma} \hat{\rho} \hat{d}_{\ell \sigma}^{\dagger}-\frac{1}{2}\left\{\hat{\rho}, \hat{d}_{\ell \sigma}^{\dagger} \hat{d}_{\ell \sigma}\right\}\right] .
$$

We take the rate $\gamma_{\ell}$ at which electrons leave via lead $\ell$ to be independent of the spin. To summarize, we work in the regime $U, \Delta \gg|V| \gg \epsilon_{\ell}, \gamma_{\ell}, \gamma_{\mathrm{CPS}}, \gamma_{\mathrm{EC}}$, where $U$ is the Coulomb interaction energy, $\Delta$ is the superconducting gap, and $V$ is the negative voltage. Because of the large negative bias, the electron transport from the QDs to the drain electrodes is unidirectional and the thermal smearing of the distribution functions in the leads becomes unimportant.

Electron waiting times.-We characterize the Cooper pair splitter by the distribution of electron waiting times. Given that an electron with spin $\sigma$ has just tunneled into lead $\ell$, the electron waiting time $\tau$ is the time that passes until another electron with spin $\sigma^{\prime}$ tunnels into lead $\ell^{\prime}$. The electron waiting time is a fluctuating quantity that must be characterized by a probability distribution. The terms in Eq. (3) of the form $\mathcal{J}_{\ell \sigma} \hat{\rho} \equiv \gamma_{\ell} \hat{d}_{\ell \sigma} \hat{\rho} \hat{d}_{\ell \sigma}^{\dagger}$ describe incoherent tunneling processes in which an electron with spin $\sigma$ in $\mathrm{QD}_{\ell}$ tunnels into lead $\ell$. The distribution of waiting times between transitions of type $i=\ell \sigma$ and $j=\ell^{\prime} \sigma^{\prime}$ can then be expressed as $[24,44,67]$

$$
\mathcal{W}_{j i}(\tau)=\frac{\operatorname{Tr}\left[\mathcal{J}_{j} e^{\left(\mathcal{L}-\mathcal{J}_{j}\right) \tau} \mathcal{J}_{i} \hat{\rho}_{S}\right]}{\operatorname{Tr}\left[\mathcal{J}_{i} \hat{\rho}_{S}\right]},
$$

where $\hat{\rho}_{S}$ is the stationary density matrix given as the normalized solution to the equation $\mathcal{L} \hat{\rho}_{S}=0$. The expression above for the WTD can be understood as follows: after a transition of type $i$ has occurred, the system is evolved until the next transition of type $j$ happens. The denominator ensures that the WTD is normalized to unity when integrated over all possible waiting times.

Figures 1(b) and 1(c) show WTDs for transitions into the same lead and different leads, respectively. Experimentally, 
transitions between different charge states can be monitored using charge detectors that measure the occupation of each QD [23,52-54]. In Fig. 1(b), we consider the waiting time between transitions into the left lead. Here, the coupling to the drain electrodes is much larger than the coupling to the superconductor, $\gamma_{L}, \gamma_{R} \gg \gamma_{\mathrm{CPS}}, \gamma_{\mathrm{EC}}$. As the QDs cannot be doubly occupied, the WTD is strongly suppressed at short times $\tau \ll \gamma_{\text {CPS }}^{-1}$ and vanishes completely at $\tau=0$, since simultaneous transitions into the same lead are not possible. At long times, the WTD is governed by the slow refilling of the left QD and the subsequent tunneling of an electron into the left lead. This WTD resembles what one would expect for single electron tunneling through a single QD without any Cooper pair splitting [24].

A very different picture emerges from the waiting time between transitions into different leads. In Fig. 1(c), the splitting of a Cooper pair is signaled by a large peak in the WTD at short times $\tau \ll \gamma_{\mathrm{CPS}}^{-1}$. In this case, the tunneling of an electron into the left lead is quickly followed by a tunneling event into the right lead on a time scale given by the coupling to the right lead $\gamma_{R}^{-1}$. The slow decay of the WTD describes the waiting time between electrons originating from different Cooper pairs. This WTD clearly reflects the nonlocal nature of the CPS processes and it carries information about the short waiting times between electrons from the same Cooper pair and the long waiting times between electrons originating from different Cooper pairs. Experimentally, a measurement of the WTD in Fig. 1(c) would constitute strong evidence of efficient Cooper pair splitting.

Exclusive WTDs.-To better understand the time scales that enter the WTDs, we introduce "exclusive" WTDs. Again, we consider the waiting time that passes between transitions of types $i$ and $j$. However, we now exclude cases, where any other transitions occur during the waiting time. This WTD is then defined as $[24,47]$

$$
\mathcal{W}_{j i}^{\mathrm{ex}}(\tau)=\frac{\operatorname{Tr}\left[\mathcal{J}_{j} e^{\mathcal{L}^{\mathrm{ex}} \tau} \mathcal{J}_{i} \hat{\rho}_{S}\right]}{\operatorname{Tr}\left[\mathcal{J}_{i} \hat{\rho}_{S}\right]}
$$

where $\mathcal{L}^{\mathrm{ex}}=\mathcal{L}-\sum_{k} \mathcal{J}_{k}$ removes all possible transitions from the full time evolution given by $\mathcal{L}$. In contrast to the WTD in Eq. (4), the exclusive WTD is only normalized upon integrating over all waiting times and summing over all types of final events. Because of its simpler structure, the exclusive WTD can be evaluated analytically. For example, with $\gamma_{L}=\gamma_{R}=\xi$ and $\epsilon_{L}=-\epsilon_{R}=\epsilon$, we find

$$
\begin{aligned}
& \mathcal{W}_{\ell \sigma, \ell^{\prime} \sigma}^{\mathrm{ex}}(\tau)=\frac{\xi}{2} e^{-\xi \tau} \alpha_{\mathrm{CPS}}^{2}\left[1-\cos \left(\omega_{\mathrm{CPS}} \tau\right)\right], \\
& \mathcal{W}_{\ell \sigma, \ell \bar{\sigma}}^{\mathrm{ex}}(\tau)=\xi e^{-\xi \tau} \alpha_{\mathrm{EC}}^{2}\left[1-\cos \left(\omega_{\mathrm{EC}} \tau\right)\right]+\mathcal{W}_{\ell \sigma, \ell \sigma}^{\mathrm{ex}}(\tau), \\
& \mathcal{W}_{\ell \sigma, \bar{\ell} \bar{\sigma}}^{\mathrm{ex}}(\tau)=\frac{\xi}{2} e^{-\xi \tau}+2 \mathcal{W}_{\ell \sigma, \ell \sigma}^{\mathrm{ex}}(\tau)-\mathcal{W}_{\ell \sigma, \ell \bar{\sigma}}^{\mathrm{ex}}(\tau),
\end{aligned}
$$

with $\bar{L}=R$ and $\bar{\uparrow}=\downarrow$ and vice versa, and we have identified the frequencies $\omega_{\mathrm{CPS}}=2 \sqrt{\gamma_{\mathrm{CPS}}^{2}-(\xi / 2)^{2}}$ and $\omega_{\mathrm{EC}}=2 \sqrt{\gamma_{\mathrm{EC}}^{2}+\epsilon^{2}}$ associated with the coherent CPS and $\mathrm{EC}$ processes and introduced the ratios $\alpha_{\mathrm{CPS}}=$ $\gamma_{\mathrm{CPS}} / \omega_{\mathrm{CPS}}$ and $\alpha_{\mathrm{EC}}=\gamma_{\mathrm{EC}} / \omega_{\mathrm{EC}}$. If $\gamma_{\mathrm{CPS}} \gg \gamma_{L}, \gamma_{R}$, the WTD exhibits oscillations with frequency $\omega_{\text {CPS }} \simeq 2 \gamma_{\text {CPS }}$. By contrast, for $\gamma_{\mathrm{CPS}} \ll \gamma_{L}, \gamma_{R}$, the frequency becomes imaginary and now rather corresponds to an exponential decay. In Fig. 1, we show the exclusive WTDs $\mathcal{W}_{\ell \ell^{\prime}}^{\text {ex }}(\tau)=\sum_{\sigma, \sigma^{\prime}} \mathcal{W}_{\ell \sigma, \ell^{\prime} \sigma^{\prime}}^{\text {ex }}(\tau) / 2$. For short times, we have $\mathcal{W}_{L L}^{\mathrm{ex}}(\tau) \sim\left(\omega_{\mathrm{CPS}} \tau\right)^{2}$. By contrast, for the WTD in Fig. 1(c) the short-time behavior $\mathcal{W}_{R L}^{\mathrm{ex}}(\tau) \sim e^{-\xi \tau}$ is governed by the escape rate, while the long-time decay $\mathcal{W}_{R L}^{\mathrm{ex}}(\tau) \sim e^{-2 \tau \gamma_{\mathrm{CP}}^{2} / \xi}$ also involves the CPS amplitude.

Spin-resolved WTDs.-The splitting of Cooper pairs can be identified in the charge-resolved WTDs as we saw in Fig. 1(c). Still, further information can be obtained from the spin-resolved WTDs. Experimentally, one might measure spin-resolved WTDs using ferromagnetic detectors [58,68-70]. In Fig. 2, we show WTDs that are resolved with respect to the spin degree of freedom. In Figs. 2(a) and 2(b), the levels are detuned so that only CPS processes are on resonance. Again, the WTDs for transitions into the same lead show essentially no signatures of the CPS processes. By contrast, the CPS processes can be identified in the WTD in Fig. 2(b) for transitions into different leads. Here, the CPS processes show up as a large enhancement at short times in the WTD for opposite spins. Because of the
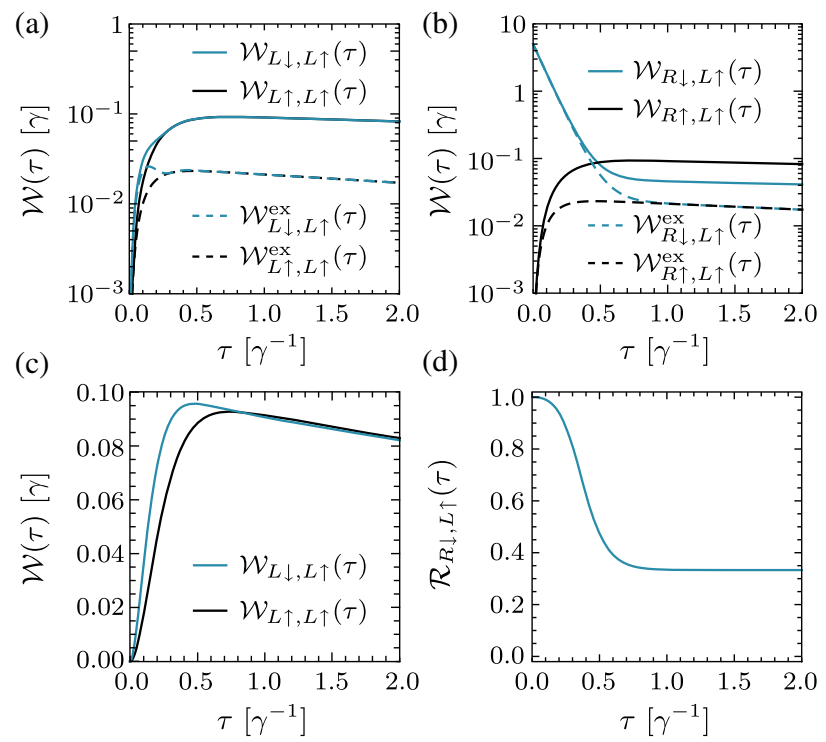

FIG. 2. Spin-resolved WTDs. (a) Spin-resolved WTDs for tunneling into the same lead. (b) Spin-resolved WTDs for tunneling into different leads. In (a) and (b), the parameters are $\gamma_{L}=\gamma_{R} \equiv 10 \gamma, \quad \gamma_{\mathrm{CPS}}=\gamma_{\mathrm{EC}}=\gamma$, and $\epsilon_{L}=-\epsilon_{R}=10 \gamma$. (c) Spin-resolved WTDs for tunneling into the same lead with same parameters except that $\epsilon_{L}=\epsilon_{R}=0$. (d) The branching ratio in Eq. (7) corresponding to the WTDs in (b). 
splitting of a Cooper pair, the tunneling of a spin-up electron into the left lead is likely followed by the tunneling of a spin-down electron into the right lead. A similar enhancement is not found for electrons with the same spin, since they must originate from different Cooper pairs.

In Fig. 2(c), both the CPS and EC processes are tuned into resonance. The combination of these processes leads to an enhancement at intermediate times in the WTD for electrons with opposite spins tunneling into the same lead. In this case, two electrons from a Cooper pair can exit into the same drain due to a spin-preserving EC process that transfers the second electron from the right to the left QD before it exits via the left drain. This is not possible for electrons with the same spin, since they cannot originate from the same Cooper pair, and the corresponding WTD is not enhanced in a similar way.

Importantly, from the spin-resolved WTDs, we can evaluate the branching ratio of the spins defined as

$$
\mathcal{R}_{R \downarrow, L \uparrow}(\tau) \equiv \frac{\mathcal{W}_{R \downarrow, L \uparrow}(\tau)}{\mathcal{W}_{R \downarrow, L \uparrow}(\tau)+\mathcal{W}_{R \uparrow, L \uparrow}(\tau)}
$$

The branching ratio is the probability that two electrons, which tunnel into different leads separated by the waiting time $\tau$, have opposite spins. Figure 2(d) shows that it is highly probable that electrons separated by a short waiting time have oppositive spins and they likely originate from the same Cooper pair. This finding is important since it allows us to conclude that the large peak in Fig. 1(c) with near-unity probability corresponds to opposite spins originating from the same Cooper pair [71].

Until now, we have assumed that the coupling to the drains is much larger than the coupling to the superconductor. This regime may be most attractive for efficient Cooper pair splitting, since the split pair of electrons is quickly transferred to the drains. However, the opposite regime, $\gamma_{\mathrm{CPS}}, \gamma_{\mathrm{EC}} \gg \gamma_{L}, \gamma_{R}$, is also interesting. In Fig. 3, the rate of escape to the drains is so slow that several coherent oscillations between the QDs and the superconductor can be completed [24,28,47]. As discussed after Eq. (6), the frequency of the oscillations is given by $\omega_{\mathrm{CPS}} \simeq 2 \gamma_{\mathrm{CPS}}$.
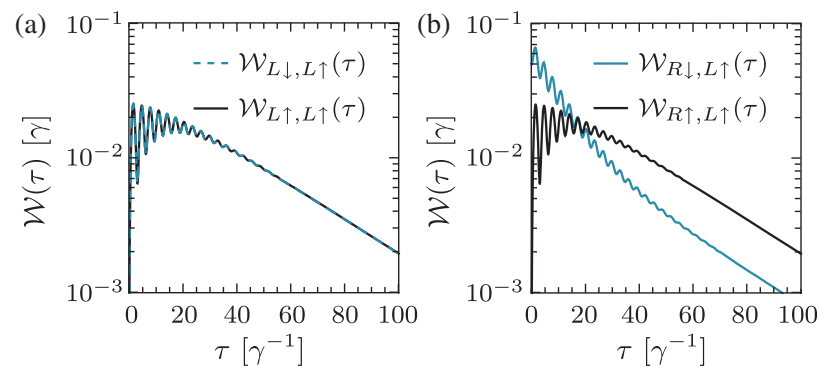

FIG. 3. Coherent oscillations. (a) Spin-resolved WTDs for tunneling into the same lead. (b) Spin-resolved WTDs for tunneling into different leads. In both (a) and (b), the parameters are $\gamma_{L}=\gamma_{R} \equiv 0.1 \gamma, \gamma_{\mathrm{CPS}}=\gamma_{\mathrm{EC}}=\gamma$, and $\epsilon_{L}=-\epsilon_{R}=10 \gamma$.
Joint WTDs.-The WTDs concern waiting times between subsequent tunneling events. However, they do not describe correlations between consecutive waiting times. Such correlations can be characterized by evaluating the joint distribution of electron waiting times $[44,49,72]$

$$
\mathcal{W}_{k j i}\left(\tau_{1}, \tau_{2}\right)=\frac{\operatorname{Tr}\left[\mathcal{J}_{k} e^{\left(\mathcal{L}-\mathcal{J}_{k}\right) \tau_{2}} \mathcal{J}_{j} e^{\left(\mathcal{L}-\mathcal{J}_{j}\right) \tau_{1}} \mathcal{J}_{i} \hat{\rho}_{S}\right]}{\operatorname{Tr}\left[\mathcal{J}_{i} \hat{\rho}_{S}\right]}
$$

which generalizes Eq. (4) to subsequent waiting times between transitions of type $i, j$, and $k$. For uncorrelated waiting times, the joint distribution factorizes as $\mathcal{W}_{k j}\left(\tau_{2}\right) \mathcal{W}_{j i}\left(\tau_{1}\right)$ in terms of the individual WTDs. Correlations can be quantified by the correlation function

$$
\Delta \mathcal{W}_{k j i}\left(\tau_{1}, \tau_{2}\right)=\frac{\mathcal{W}_{k j i}\left(\tau_{1}, \tau_{2}\right)-\mathcal{W}_{k j}\left(\tau_{2}\right) \mathcal{W}_{j i}\left(\tau_{1}\right)}{\mathcal{W}_{k j}\left(\tau_{2}\right) \mathcal{W}_{j i}\left(\tau_{1}\right)}
$$

which is positive (negative) for positively (negatively) correlated waiting times and zero without correlations.

Figure 4 shows joint WTDs and correlation functions for electrons arriving in different leads. In Figs. 4(a) and 4(b), the coupling to the drains is much larger than the coupling to the superconductor. We see that a short waiting time is likely followed by a long waiting time, but is unlikely to be followed by another short waiting time. A short waiting time corresponds to two electrons originating from the same Cooper pair, while a long waiting time is given by the slow refilling of the QDs by a split Cooper pair. The observed correlations reflect that the two processes, i.e., emission into the drains and refilling from the
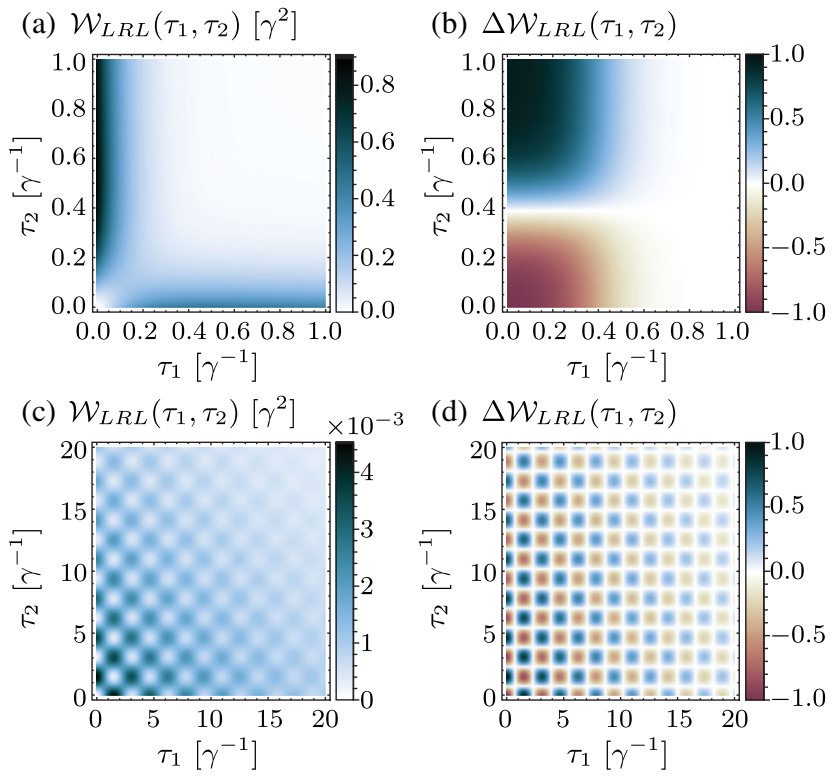

(d) $\Delta \mathcal{W}_{L R L}\left(\tau_{1}, \tau_{2}\right)$

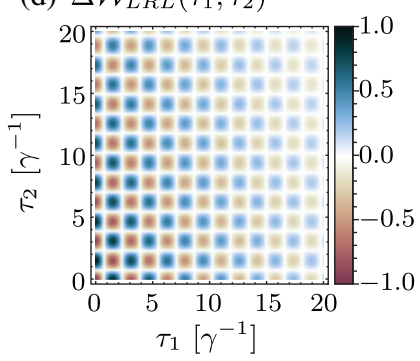

FIG. 4. Joint WTDs and correlation functions. In (a) and (b), the parameters are $\gamma_{L}=\gamma_{R}=10 \gamma, \quad \gamma_{\mathrm{CPS}}=\gamma_{\mathrm{EC}} \equiv \gamma$, and $\epsilon_{L}=\epsilon_{R}=0$. In (c) and (d), the parameters are $\gamma_{L}=\gamma_{R}=0.1 \gamma$, $\gamma_{\mathrm{CPS}}=\gamma_{\mathrm{EC}}=\gamma$, and $\epsilon_{L}=\epsilon_{R}=0$. 
superconductor, follow one after another. A similar behavior is seen in Figs. 4(c) and 4(d), where the coupling to the superconductor is the largest. However, now the rate of escape to the drains is so slow that coherent oscillations between the QDs and the superconductor have time to form, giving rise to the oscillatory pattern in the joint WTD and the correlation function.

Conclusions. - We have proposed to use waiting time distributions to characterize Cooper pair splitters. The nonlocal nature of the Cooper pair splitting can be clearly identified in the distribution of waiting times. Based on the recent progress in the real-time detection of Andreev tunneling, we expect the predictions to be accessible in future experiments. Specifically, a measurement of the WTD would constitute a strong evidence of efficient Cooper pair splitting in the regime of slow tunneling, where conventional current measurements are demanding. Theoretically, it would be interesting to formulate a Belllike inequality for the waiting times to certify the entanglement of the split Cooper pairs.

We thank P. J. Hakonen, G. B. Lesovik, M. V. Moskalets, J. P. Pekola, and B. Sothmann for valuable discussions. Authors at Aalto are associated with Centre for Quantum Engineering. The Center for Nanostructured Graphene is sponsored by the Danish National Research Foundation (Project No. DNRF103). The work was supported by the Academy of Finland through its Centre of Excellence program (Project No. 312299).

[1] A. Zagoskin, Quantum engineering: Theory and design of quantum coherent structures (Cambridge University Press, Cambridge, England, 2011).

[2] T. D. Ladd, F. Jelezko, R. Laflamme, Y. Nakamura, C. Monroe, and J. L. O'Brien, Review: Quantum computers, Nature (London) 464, 45 (2010).

[3] G. B. Lesovik, T. Martin, and G. Blatter, Electronic entanglement in the vicinity of a superconductor, Eur. Phys. J. B 24, 287 (2001).

[4] P. Recher, E. V. Sukhorukov, and D. Loss, Andreev tunneling, Coulomb blockade, and resonant transport of nonlocal spin-entangled electrons, Phys. Rev. B 63, 165314 (2001).

[5] D. Beckmann, H. B. Weber, and H. v. Löhneysen, Evidence for Crossed Andreev Reflection in SuperconductorFerromagnet Hybrid Structures, Phys. Rev. Lett. 93, 197003 (2004).

[6] S. Russo, M. Kroug, T. M. Klapwijk, and A. F. Morpurgo, Experimental Observation of Bias-Dependent Nonlocal Andreev Reflection, Phys. Rev. Lett. 95, 027002 (2005).

[7] R. S. Deacon, A. Oiwa, J. Sailer, S. Baba, Y. Kanai, K. Shibata, K. Hirakawa, and S. Tarucha, Cooper pair splitting in parallel quantum dot Josephson junctions, Nat. Commun. 6, 7446 (2015).

[8] L. Hofstetter, S. Csonka, J. Nygård, and C. Schönenberger, Cooper pair splitter realized in a two-quantum-dot Y-junction, Nature (London) 461, 960 (2009).
[9] L. Hofstetter, S. Csonka, A. Baumgartner, G. Fülöp, S. d'Hollosy, J. Nygård, and C. Schönenberger, Finite-Bias Cooper Pair Splitting, Phys. Rev. Lett. 107, 136801 (2011).

[10] A. Das, R. Ronen, M. Heiblum, D. Mahalu, A. V. Kretinin, and H. Shtrikman, High-efficiency Cooper pair splitting demonstrated by two-particle conductance resonance and positive noise cross-correlation, Nat. Commun. 3, 1165 (2012).

[11] G. Fülöp, F. Domínguez, S. d'Hollosy, A. Baumgartner, P. Makk, M. H. Madsen, V. A. Guzenko, J. Nygård, C. Schönenberger, A. Levy Yeyati, and S. Csonka, Magnetic Field Tuning and Quantum Interference in a Cooper Pair Splitter, Phys. Rev. Lett. 115, 227003 (2015).

[12] L. G. Herrmann, F. Portier, P. Roche, A. L. Yeyati, T. Kontos, and C. Strunk, Carbon Nanotubes as Cooper-Pair Beam Splitters, Phys. Rev. Lett. 104, 026801 (2010).

[13] L. G. Herrmann, P. Burset, W. J. Herrera, F. Portier, P. Roche, C. Strunk, A. Levy Yeyati, and T. Kontos, Spectroscopy of non-local superconducting correlations in a double quantum dot, arXiv:1205.1972.

[14] J. Schindele, A. Baumgartner, and C. Schönenberger, NearUnity Cooper Pair Splitting Efficiency, Phys. Rev. Lett. 109, 157002 (2012).

[15] G. Fülöp, S. d'Hollosy, A. Baumgartner, P. Makk, V. A. Guzenko, M. H. Madsen, J. Nygård, C. Schönenberger, and S. Csonka, Local electrical tuning of the nonlocal signals in a Cooper pair splitter, Phys. Rev. B 90, 235412 (2014).

[16] J. Schindele, A. Baumgartner, R. Maurand, M. Weiss, and C. Schönenberger, Nonlocal spectroscopy of Andreev bound states, Phys. Rev. B 89, 045422 (2014).

[17] Z. B. Tan, D. Cox, T. Nieminen, P. Lähteenmäki, D. Golubev, G. B. Lesovik, and P. J. Hakonen, Cooper Pair Splitting by Means of Graphene Quantum Dots, Phys. Rev. Lett. 114, 096602 (2015).

[18] I. V. Borzenets, Y. Shimazaki, G. F. Jones, M. F. Craciun, S. Russo, M. Yamamoto, and S. Tarucha, High efficiency CVD graphene-lead $(\mathrm{Pb})$ Cooper pair splitter, Sci. Rep. 6, 23051 (2016).

[19] SK Firoz Islam and A. Saha, Amplification of Cooper pair splitting current in a graphene-based Cooper pair beam splitter geometry, Phys. Rev. B 96, 125406 (2017).

[20] S. Kawabata, Test of Bell's inequality using the spin filter effect in ferromagnetic semiconductor microstructures, J. Phys. Soc. Jpn. 70, 1210 (2001).

[21] N. M. Chtchelkatchev, G. Blatter, G. B. Lesovik, and T. Martin, Bell inequalities and entanglement in solid-state devices, Phys. Rev. B 66, 161320 (2002).

[22] O. Sauret, T. Martin, and D. Feinberg, Spin-current noise and Bell inequalities in a realistic superconductor-quantum dot entangler, Phys. Rev. B 72, 024544 (2005).

[23] S. Gustavsson, R. Leturcq, M. Studer, I. Shorubalko, T. Ihn, K. Ensslin, D. C. Driscoll, and A.C. Gossard, Electron counting in quantum dots, Surf. Sci. Rep. 64, 191 (2009).

[24] T. Brandes, Waiting times and noise in single particle transport, Ann. Phys. (Berlin) 17, 477 (2008).

[25] S. Welack, M. Esposito, U. Harbola, and S. Mukamel, Interference effects in the counting statistics of electron transfers through a double quantum dot, Phys. Rev. B 77, 195315 (2008). 
[26] S. Welack, S. Mukamel, and Y. J. Yan, Waiting time distributions of electron transfers through quantum dot AharonovBohm interferometers, Europhys. Lett. 85, 57008 (2009).

[27] M. Albert, C. Flindt, and M. Büttiker, Distributions of Waiting Times of Dynamic Single-Electron Emitters, Phys. Rev. Lett. 107, 086805 (2011).

[28] K. H. Thomas and C. Flindt, Electron waiting times in nonMarkovian quantum transport, Phys. Rev. B 87, 121405 (2013).

[29] G.-M. Tang, F. Xu, and J. Wang, Waiting time distribution of quantum electronic transport in the transient regime, Phys. Rev. B 89, 205310 (2014).

[30] G.-M. Tang and J. Wang, Full-counting statistics of charge and spin transport in the transient regime: A nonequilibrium Green's function approach, Phys. Rev. B 90, 195422 (2014).

[31] B. Sothmann, Electronic waiting-time distribution of a quantum-dot spin valve, Phys. Rev. B 90, 155315 (2014).

[32] R. Seoane Souto, R. Avriller, R. C. Monreal, A. MartínRodero, and A. Levy Yeyati, Transient dynamics and waiting time distribution of molecular junctions in the polaronic regime, Phys. Rev. B 92, 125435 (2015).

[33] V. Talbo, J. Mateos, S. Retailleau, P. Dollfus, and T. González, Time-dependent shot noise in multi-level quantum dot-based single-electron devices, Semicond. Sci. Technol. 30, 055002 (2015).

[34] S. L. Rudge and D. S. Kosov, Distribution of residence times as a marker to distinguish different pathways for quantum transport, Phys. Rev. E 94, 042134 (2016).

[35] S. L. Rudge and D. S. Kosov, Distribution of tunnelling times for quantum electron transport, J. Chem. Phys. 144, 124105 (2016).

[36] K. Ptaszyński, Nonrenewal statistics in transport through quantum dots, Phys. Rev. B 95, 045306 (2017).

[37] E. Potanina and C. Flindt, Electron waiting times of a periodically driven single-electron turnstile, Phys. Rev. B 96, 045420 (2017).

[38] D. S. Kosov, Non-renewal statistics for electron transport in a molecular junction with electron-vibration interaction, J. Chem. Phys. 147, 104109 (2017).

[39] M. Albert, G. Haack, C. Flindt, and M. Büttiker, Electron Waiting Times in Mesoscopic Conductors, Phys. Rev. Lett. 108, 186806 (2012).

[40] G. Haack, M. Albert, and C. Flindt, Distributions of electron waiting times in quantum-coherent conductors, Phys. Rev. B 90, 205429 (2014).

[41] K. H. Thomas and C. Flindt, Waiting time distributions of noninteracting fermions on a tight-binding chain, Phys. Rev. B 89, 245420 (2014).

[42] D. Dasenbrook, C. Flindt, and M. Büttiker, Floquet Theory of Electron Waiting Times in Quantum-Coherent Conductors, Phys. Rev. Lett. 112, 146801 (2014).

[43] M. Albert and P. Devillard, Waiting time distribution for trains of quantized electron pulses, Phys. Rev. B 90, 035431 (2014).

[44] D. Dasenbrook, P. P. Hofer, and C. Flindt, Electron waiting times in coherent conductors are correlated, Phys. Rev. B 91, 195420 (2015).

[45] D. Dasenbrook and C. Flindt, Quantum theory of an electron waiting time clock, Phys. Rev. B 93, 245409 (2016).
[46] P. P. Hofer, D. Dasenbrook, and C. Flindt, Electron waiting times for the mesoscopic capacitor, Physica (Berlin) 82E, 3 (2016).

[47] L. Rajabi, C. Pöltl, and M. Governale, Waiting Time Distributions for the Transport through a Quantum-Dot Tunnel Coupled to One Normal and One Superconducting Lead, Phys. Rev. Lett. 111, 067002 (2013).

[48] S. Dambach, B. Kubala, V. Gramich, and J. Ankerhold, Time-resolved statistics of nonclassical light in Josephson photonics, Phys. Rev. B 92, 054508 (2015).

[49] S. Dambach, B. Kubala, and J. Ankerhold, Time-resolved statistics of photon pairs in two-cavity Josephson photonics, Fortschr. Phys. 65, 1600061 (2017).

[50] M. Albert, D. Chevallier, and P. Devillard, Waiting times of entangled electrons in normal-superconducting junctions, Physica (Amsterdam) 76E, 209 (2016).

[51] D. Chevallier, M. Albert, and P. Devillard, Probing Majorana and Andreev bound states with waiting times, Europhys. Lett. 116, 27005 (2016).

[52] S. K. Gorman, Y. He, M. G. House, J. G. Keizer, D. Keith, L. Fricke, S. J. Hile, M. A. Broome, and M. Y. Simmons, Tunneling Statistics for Analysis of Spin-Readout Fidelity, Phys. Rev. Applied 8, 034019 (2017).

[53] V. F. Maisi, O.-P. Saira, Yu. A. Pashkin, J. S. Tsai, D. V. Averin, and J. P. Pekola, Real-Time Observation of Discrete Andreev Tunneling Events, Phys. Rev. Lett. 106, 217003 (2011).

[54] V. F. Maisi, D. Kambly, C. Flindt, and J. P. Pekola, Full Counting Statistics of Andreev Tunneling, Phys. Rev. Lett. 112, 036801 (2014).

[55] O. Sauret, D. Feinberg, and T. Martin, Quantum master equations for the superconductor-quantum dot entangler, Phys. Rev. B 70, 245313 (2004).

[56] A. Braggio, M. Governale, M. G. Pala, and J. König, Superconducting proximity effect in interacting quantum dots revealed by shot noise, Solid State Commun. 151, 155 (2011).

[57] J. Eldridge, M. G. Pala, M. Governale, and J. König, Superconducting proximity effect in interacting doubledot systems, Phys. Rev. B 82, 184507 (2010).

[58] P. Trocha and I. Weymann, Spin-resolved Andreev transport through double-quantum-dot Cooper pair splitters, Phys. Rev. B 91, 235424 (2015).

[59] E. Amitai, R. P. Tiwari, S. Walter, T. L. Schmidt, and S. E. Nigg, Nonlocal quantum state engineering with the Cooper pair splitter beyond the Coulomb blockade regime, Phys. Rev. B 93, 075421 (2016).

[60] R. Hussein, L. Jaurigue, M. Governale, and A. Braggio, Double quantum dot Cooper-pair splitter at finite couplings, Phys. Rev. B 94, 235134 (2016).

[61] R. Hussein, A. Braggio, and M. Governale, Entanglementsymmetry control in a quantum-dot Cooper-pair splitter, Phys. Status Solidi B 254, 1600603 (2017).

[62] D. Chevallier, J. Rech, T. Jonckheere, and T. Martin, Current and noise correlations in a double-dot Cooper-pair beam splitter, Phys. Rev. B 83, 125421 (2011).

[63] B. Hiltscher, M. Governale, J. Splettstoesser, and J. König, Adiabatic pumping in a double-dot Cooper-pair beam splitter, Phys. Rev. B 84, 155403 (2011). 
[64] M. Flöser, D. Feinberg, and R. Mélin, Absence of split pairs in cross correlations of a highly transparent normal metalsuperconductor-normal metal electron-beam splitter, Phys. Rev. B 88, 094517 (2013).

[65] I. A. Sadovskyy, G. B. Lesovik, and V. M. Vinokur, Unitary limit in crossed Andreev transport, New J. Phys. 17, 103016 (2015).

[66] Sh. Gurvitz, Wave-function approach to Master equations for quantum transport and measurement, Front. Phys. 12, 120303 (2017).

[67] H. J. Carmichael, S. Singh, R. Vyas, and P. R. Rice, Photoelectron waiting times and atomic state reduction in resonance fluorescence, Phys. Rev. A 39, 1200 (1989).

[68] O. Malkoc, C. Bergenfeldt, and P. Samuelsson, Full counting statistics of generic spin entangler with quantum dot-ferromagnet detectors, Europhys. Lett. 105, 47013 (2014).

[69] P. Busz, D. Tomaszewski, and J. Martinek, Spin correlation and entanglement detection in Cooper pair splitters by current measurements using magnetic detectors, Phys. Rev. B 96, 064520 (2017).

[70] K. Wrześniewski, P. Trocha, and I. Weymann, Current cross-correlations in double quantum dot based Cooper pair splitters with ferromagnetic leads, J. Phys. Condens. Matter 29, 195302 (2017).

[71] Z. Scherübl, A. Pályi, and S. Csonka, Probing individual split Cooper pairs using the spin qubit toolkit, Phys. Rev. B 89, 205439 (2014).

[72] X. H. H. Zhang and H. U. Baranger, Photon Statistics in a Waveguide: Beyond Bunching and Antibunching, arXiv: 1710.01543 . 